\title{
Examination of hospital length of stay in Canada among patients with acute bacterial skin and skin structure infection caused by methicillin-resistant Staphylococcus aureus
}

\author{
This article was published in the following Dove Press journal: \\ Infection and Drug Resistance \\ 28 January 2016 \\ Number of times this article has been viewed
}

\section{Michele H Potashman' \\ Michael Stokes ${ }^{2}$ \\ Jieruo Liu² \\ Robin Lawrence' \\ Linda Harris'}

'Global Health Outcomes, Merck \& Co, Inc., Kenilworth, NJ, USA; ${ }^{2}$ Evidera, Lexington, MA, USA
Correspondence: Linda Harris Global Health Outcomes, Merck \& Co, Inc., 2000 Galloping Hill Road, Kenilworth, NJ 07033, USA

Tel +l 9087404000

Email lindah303।@gmail.com
Purpose: Skin infections, particularly those caused by resistant pathogens, represent a clinical burden. Hospitalization associated with acute bacterial skin and skin structure infections (ABSSSI) caused by methicillin-resistant Staphylococcus aureus (MRSA) is a major contributor to the economic burden of the disease. This study was conducted to provide current, real-world data on hospitalization patterns for patients with ABSSSI caused by MRSA across multiple geographic regions in Canada.

Patients and methods: This retrospective cohort study evaluated length of stay (LOS) for hospitalized patients with ABSSSI due to MRSA diagnosis across four Canadian geographic regions using the Discharge Abstract Database. Patients with ICD-10-CA diagnosis consistent with ABSSSI caused by MRSA between January 2008 and December 2014 were selected and assigned a primary or secondary diagnosis based on a prespecified ICD-10-CA code algorithm.

Results: Among 6,719 patients, 3,273 (48.7\%) and 3,446 (51.3\%) had a primary and secondary diagnosis, respectively. Among patients with a primary or secondary diagnosis, the cellulitis/erysipelas subtype was most common. The majority of patients presented with 0 or 1 comorbid condition; the most common comorbidity was diabetes. The mean LOS over the study period varied by geographic region and year; in 2014 (the most recent year analyzed), LOS ranged from 7.7 days in Ontario to 13.4 days in the Canadian Prairie for a primary diagnosis and from 18.2 days in Ontario to 25.2 days in Atlantic Canada for a secondary diagnosis. A secondary diagnosis was associated with higher rates of continuing care compared with a primary diagnosis $(10.6 \%-24.2 \%$ vs $4.6 \%-12.1 \%)$.

Conclusion: This study demonstrated that the mean LOS associated with ABSSSI due to MRSA in Canada was minimally 7 days. Clinical management strategies, including medication management, which might facilitate hospital discharge, have the potential to reduce hospital LOS and related economic burden associated with ABSSSI caused by MRSA.

Keywords: ABSSSI, MRSA, Canadian provinces, LOS, hospital outcomes, time trends

\section{Introduction}

Infections of the skin, particularly those caused by resistant pathogens, represent a clinical challenge to address. Acute bacterial skin and skin structure infections (ABSSSI) are a new classification of skin and skin structure infections (SSSIs) in accordance with Health Canada, the US Food and Drug Administration, and the European Medicines Commission. ABSSSI include cellulitis/erysipelas, wound infection, and major cutaneous abscess with a minimum lesion surface area of $75 \mathrm{~cm}^{2} .{ }^{1}$ ABSSSI is one of several 
classifications of skin infections (skin and soft tissue infection [SSTI], complicated SSSI [cSSSI], and complicated SSTI [cSSTI]). A key distinction of ABSSSI from other classifications is that it excludes less serious skin infections as well as infections needing more complex treatment regimens.

As ABSSSI is a recent classification, limited literature exists to inform the burden of illness for this patient population. However, several authors have reported data for SSTI, SSSI, and cSSTI, and, although these reports are for skin infections with a broader population base than ABSSSI alone, the trends are informative for ABSSSI patients. SSTIs are most frequently caused by Gram-positive pathogens, with methicillin-resistant Staphylococcus aureus (MRSA) being increasingly the predominant pathogen in the USA and Europe..$^{2-5}$ Linked to the growing prevalence of MRSA, rates of hospitalization for SSTI have increased in the last decade in the USA.${ }^{6,7}$ Reports from Canada also highlight the increasing prevalence of SSTI due to MRSA in outpatients, emergency departments (ED), and hospitals. ${ }^{8-13}$ In a study of visits to the ED for SSTI, the prevalence of MRSA varied significantly across the Canadian provinces (11\%-100\%), with a higher prevalence in Western Canada. ${ }^{12}$ Data from the Canadian Nosocomial Infection Surveillance Program from 1995 to 2007 showed that hospitalization for SSTI caused by MRSA increased from 24\% to 37\% between 1994 and 2007. ${ }^{13}$ Surveillance reports have also documented increased isolation of MRSA from hospitalized patients with SSTI in North America, although rates were lower in Canada than in the USA. ${ }^{2,14}$

The involvement of MRSA in skin infections adversely affects patient outcomes. Skin infections caused by MRSA are associated with increased instances of treatment failure, ${ }^{15-17}$ disease recurrence, ${ }^{16,18}$ increased risk of invasive disease, ${ }^{19}$ and higher mortality. ${ }^{15,20-22}$ MRSA-positive skin infections also have a significant economic impact because of increased durations of hospitalization ${ }^{15,22,23}$ and higher hospital costs. ${ }^{15}$

Real-world data on cost drivers in patients with skin infections due to MRSA are important, so that health care can be delivered effectively and efficiently in an increasingly cost-constrained environment. Hospitalization is the major cost driver associated with MRSA infection. ${ }^{24,25}$ Hospital length of stay (LOS) is an important outcomes measure for patients with cSSTI due to MRSA, as the majority of medical costs for this condition are related to hospital ward and intensive care unit (ICU) stays. ${ }^{26,27}$ Although SSTI are among the most common infections necessitating hospitalization, ${ }^{6}$ in general, there is limited countrywide information on hospi- talizations for patients with skin infections caused by MRSA. Some data have been reported for Europe and the USA. A retrospective medical chart review of patients hospitalized with cSSTI between 2010 and 2011 in 12 European countries showed a mean hospital LOS of 20.6 days. ${ }^{28}$ In the USA, a retrospective multicenter observational registry of patients with MRSA skin infections treated with daptomycin showed median antibiotic-related LOS of 4-8 days, depending on age and comorbidities. ${ }^{29}$ Another large US database study that used 4-year hospital discharge data from the Solucient database documented mean and median LOS for cSSTI due to MRSA of 12.6 (standard deviation [SD]: 18.9) days and 7.0 days, respectively. ${ }^{21}$ In patients hospitalized with cSSTI due to infection with $S$. aureus, Healthcare Cost and Utilization Project (HCUP) data for 2009 showed an LOS of 7.3 days, ${ }^{7}$ and another US study using the Premier Perspective database reported an average hospital LOS of 6.1 days for the 2005-2006 period. $^{30}$

Canadian data on ABSSSI due to MRSA are limited. In 2003, a small study of 89 patients with SSTI due to MRSA reported a mean hospital LOS of 28.9 (SD: 29.8) days in patients treated with intravenous (IV) vancomycin. ${ }^{31}$ A large retrospective database study conducted over a 5-year period (2004 through 2008 inclusive) in $\sim 65,000$ patients hospitalized for cellulitis (not MRSA-specific) in Canada found a mean hospital LOS of 7.1 (SD: 8.2) days; $29.5 \%$ of patients admitted for cellulitis experienced a prolonged LOS in hospital ( $>7$ days). ${ }^{32}$

To narrow the information gap for Canada, we conducted a retrospective database analysis to characterize a cohort of patients hospitalized with ABSSSI due to MRSA across multiple provinces (Alberta, British Columbia, Manitoba, Newfoundland, New Brunswick, Nova Scotia, Ontario, Prince Edward Island, and Saskatchewan) grouped into four geographic regions. Demographic and clinical features, LOS, treatment patterns, and time trends were analyzed.

\section{Patients and methods}

\section{Data source}

The study used inpatient records from the Discharge Abstract Database (DAD). The DAD is a national database containing information primarily related to hospital inpatient events; the records represent $75 \%$ of all patient discharges in Canada (not including Quebec). The DAD collects, processes, and analyzes all discharges from acute care inpatient facilities, day surgery procedures, long-term care, and rehabilitation and psychiatry facilities, and contains data from 1979 to 2014. The database includes demographic information, calculated 
LOS, admission and discharge dates, most responsible diagnosis (this refers to the condition responsible for the longest LOS [ICD-10-CA coding system]), up to ten secondary diagnoses (secondary diagnoses included codes for preexisting, post-admitting, and admitting diagnoses), and diagnostic and therapeutic procedures (Canadian Classification of Interventions [CCI] coding system). Information within the DAD is de-identified and maintains full compliance with the Health Insurance Portability and Accountability Act regulations; therefore, because this study was a retrospective database study using de-identified patient information, it is exempt from IRB approval per regulatory guidance.

\section{Patient selection}

Patients were included if they were 18 years of age and older and were hospitalized between January 1, 2008 and December 31, 2014, with ICD-10-CA diagnosis codes consistent with ABSSSI due to MRSA diagnosis (see Table S1 for ABSSSI codes). The ICD-10-CA codes selected were consistent with the codes used for the three ABSSSI infection types described in the ABSSSI FDA ${ }^{1}$ definition and were cross-validated with those used in a retrospective population-based study in Canada. ${ }^{32}$ They were also checked for consistency by medical personnel and Canadian Institute for Health Information (CIHI) analysts. Indications that are not part of the ABSSSI domains with codes indicative of a more chronic condition were excluded from the analysis. Of note, although there was no way of obtaining the size of the lesion, the approach described earlier was an efficient way to identify the ABSSSI patient population in the absence of clinical data.

The patient was assumed to have ABSSSI caused by MRSA if both codes B95.6 and U82.1 were present in the hospital record, in addition to codes for ABSSSI. Data from patients' initial (or index) ABSSSI due to MRSA hospitalization were analyzed. To create an operational definition that represented acute and newly diagnosed disease, patients were excluded if their ICD-10-CA codes indicated hospitalization for MRSA infection during the 30 -day period preceding the index hospitalization. To reduce the potential for confounding due to coinfection, patients were excluded if they had any of the following coexisting conditions: pneumonia, septic (pyogenic) arthritis, neutropenia, bacteremia, and intra-abdominal infections, including appendicitis, diverticular disease with perforation and abscess, peritonitis, and cholecystitis, as identified by the ICD-10-CA diagnosis codes (any position) on the index hospitalization record.
Patients were categorized into two subgroups depending on whether ABSSSI due to MRSA was the primary or the secondary diagnosis. Patients were assigned to the primary diagnosis group if they had at least one ICD-10-CA code indicating either ABSSSI or MRSA as the most responsible diagnosis on their hospital claim. Patients were assigned to the secondary diagnosis group if the ICD-10-CA codes were present for both ABSSSI and MRSA as a secondary diagnosis and if there was at least one code for another condition or surgery coded as the most responsible diagnosis.

\section{Study measures and outcomes}

Demographic parameters analyzed were age and age-group (both as of the index hospitalization), age distribution, sex, year of index ABSSSI due to MRSA hospitalization, type of admission (emergent or elective), and the responsible payer for hospital charges (resident province/territory, other province/territory, Canadian resident self-pay, other countries resident self-pay, and other). Clinical characteristics included the ABSSSI caused by MRSA subtype (cellulitis/erysipelas, major or deep abscess, wound infection, and other [pyoderma, pyoderma gangrenosum, and pyogenic granuloma]), anatomical site of infection (limb, trunk, head/neck/face, or multiple), major comorbid conditions (these included important prevalent conditions that could impact medical resource use), and number of comorbidities.

The main outcome measure was LOS for the index ABSSSI due to MRSA admission, which was calculated from dates of admission and discharge on the hospital record. Secondary outcomes were LOS in a specialty care facility (nursing homes, residences for senior citizens, and chronic and long-term care and related facilities), main patient service (general medicine, dermatology, general surgery, other surgery, infectious diseases) that contributed to the longest portion of the patient's LOS, discharge status (died in hospital, discharged to home, continuing care [defined as ongoing care delivered to patients not ready for hospital discharge, either in a separate facility or co located with acute services in the hospital]), administration of antibiotics, and surgical procedures (incision, drainage, surgical debridement, wound excision, and amputation). An exploratory analysis of time trends for LOS was conducted for each geographic region.

\section{Data analysis}

Descriptive statistics were used to summarize baseline patient characteristics, treatment patterns, and study outcomes, including LOS for each patient subgroup. Mean, SD, median, interquartile range, and minimum and maximum values were 
reported for continuous variables. Frequency distribution with percentages was reported for categorical variables. Analyses were separately conducted for four specific regions of Canada (British Columbia/Alberta, Ontario, Canadian Prairie [Manitoba and Saskatchewan], and Atlantic Canada [Newfoundland, New Brunswick, Nova Scotia, and Prince Edward Island]). Analyses of LOS, use of surgical procedures, and main patient service utilization were performed according to the hospitalization year to examine time trends. Missing data are reported for all applicable study measures.

\section{Results}

\section{Patient population}

Figure 1 shows the selection of patients from the database. Among the 6,719 patients included in the study, 3,273 (48.7\%) had ABSSSI due to MRSA as the primary reason for the hospital stay and 3,446 (51.3\%) had ABSSSI due to MRSA as the secondary reason for the hospital stay.

Tables 1-3 summarize the demographic, clinical, and hospitalization characteristics of patients across all the geographic regions. The sample showed a slightly higher proportion of males. The mean age of patients hospitalized with a primary diagnosis ranged from 49 to 55 years, while the mean age for patients with a secondary diagnosis ranged from 55 to 67 years, indicating that patients with a secondary diagnosis were older than those with a primary diagnosis (Table 1). For all provinces except Atlantic Canada, patients aged 55 years and older were more likely to experience ABSSSI as a result of MRSA as a secondary diagnosis. In Atlantic Canada, there were 446 vs 173 patients hospitalized with a secondary vs primary diagnosis, respectively. In specifically analyzing patients aged 65 years or older from

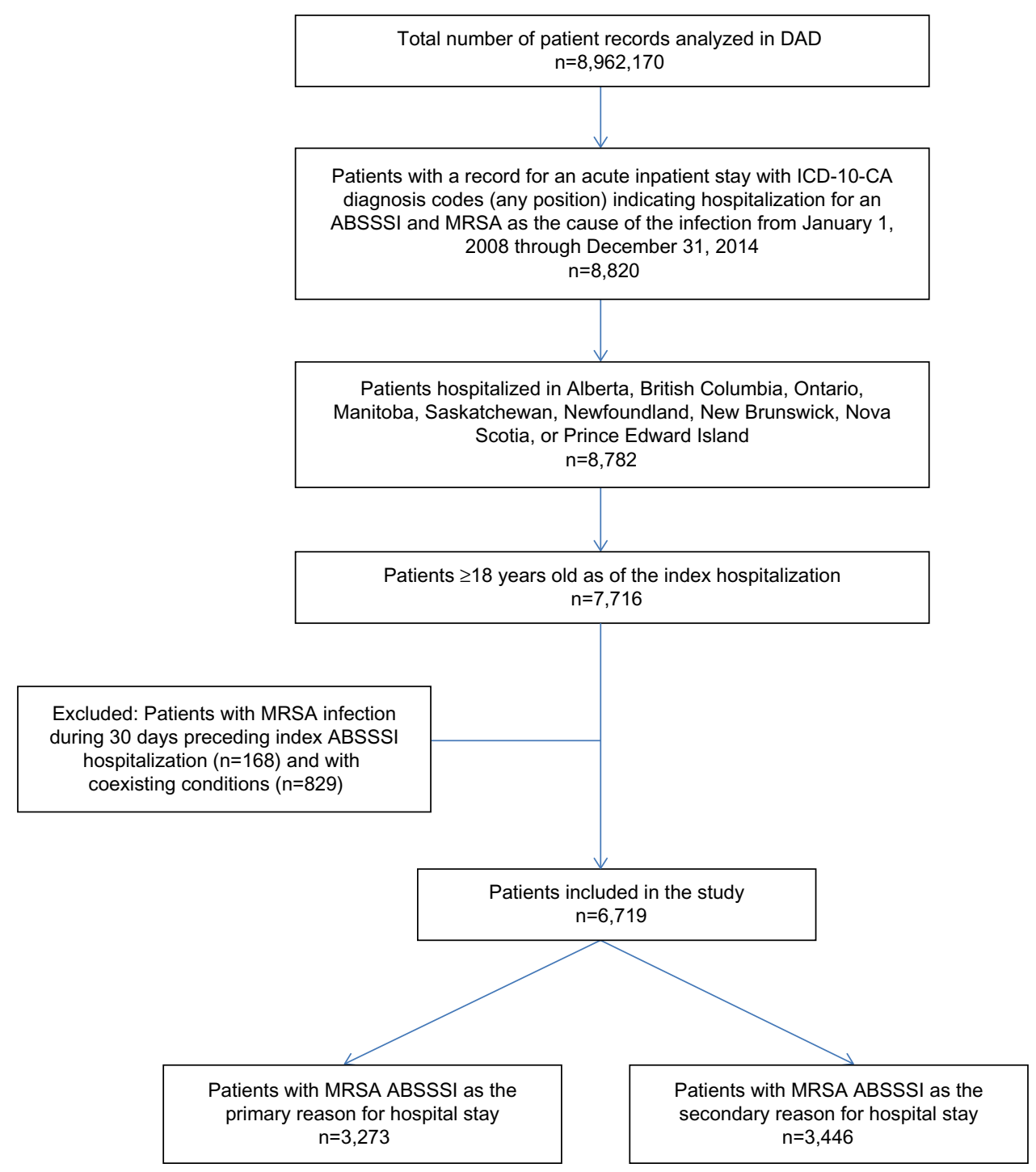

Figure I Flow diagram of sample selection and classification.

Abbreviations: ABSSSI, acute bacterial skin and skin structure infections; MRSA, methicillin-resistant Staphylococcus aureus; DAD, Discharge Abstract Database. 
Table I Demographics by province

\begin{tabular}{|c|c|c|c|c|c|c|c|c|}
\hline \multirow[t]{2}{*}{ Characteristic } & \multicolumn{2}{|l|}{ Ontario } & \multicolumn{2}{|c|}{$\begin{array}{l}\text { Alberta and British } \\
\text { Columbia }\end{array}$} & \multicolumn{2}{|c|}{ Canadian Prairie } & \multicolumn{2}{|c|}{ Atlantic Canada } \\
\hline & $\begin{array}{l}\text { Primary } \\
(\mathrm{N}=1,023)\end{array}$ & $\begin{array}{l}\text { Secondary } \\
(\mathrm{N}=953)\end{array}$ & $\begin{array}{l}\text { Primary } \\
(\mathrm{N}=1,602)\end{array}$ & $\begin{array}{l}\text { Secondary } \\
(N=1,670)\end{array}$ & $\begin{array}{l}\text { Primary } \\
(N=475)\end{array}$ & $\begin{array}{l}\text { Secondary } \\
(N=377)\end{array}$ & $\begin{array}{l}\text { Primary } \\
(\mathrm{N}=173)\end{array}$ & $\begin{array}{l}\text { Secondary } \\
(\mathrm{N}=446)\end{array}$ \\
\hline \multicolumn{9}{|l|}{ Age group, n (\%) } \\
\hline I8-44 years & $379(37)$ & I4I (I4.8) & $618(38.6)$ & $380(22.8)$ & $225(47.4)$ & $127(33.7)$ & $54(3 \mid .2)$ & $45(10.1)$ \\
\hline $45-54$ years & $186(18.2)$ & $116(12.2)$ & $308(19.2)$ & $287(17.2)$ & $94(19.8)$ & $56(14.9)$ & $28(16.2)$ & $47(10.5)$ \\
\hline $55-64$ years & $137(13.4)$ & $185(19.4)$ & $217(13.5)$ & $296(17.7)$ & $61(12.8)$ & $70(18.6)$ & $43(24.9)$ & $87(19.5)$ \\
\hline $65-74$ years & $118(11.5)$ & $194(20.4)$ & $172(10.7)$ & $264(15.8)$ & $44(9.3)$ & $61(16.2)$ & $18(10.4)$ & $104(23.3)$ \\
\hline$\geq 75$ years & $203(19.8)$ & $317(33.3)$ & $287(17.9)$ & $443(26.5)$ & $5 I(10.7)$ & $63(16.7)$ & $30(17.3)$ & $163(36.5)$ \\
\hline \multicolumn{9}{|l|}{ Age, years } \\
\hline Mean & 54 & 65 & 53 & 60 & 49 & 55 & 55 & 67 \\
\hline Standard deviation & 20 & 18 & 20 & 19 & 19 & 20 & 19 & 17 \\
\hline Median & 52 & 66 & 50 & 60 & 45 & 56 & 56 & 69 \\
\hline IQR & $38-70$ & $53-79$ & $38-68$ & $46-77$ & $36-59$ & $40-70$ & $4 I-66$ & $57-81$ \\
\hline Range & $18-103$ & $19-103$ & $|8-10|$ & $18-105$ & $|8-10|$ & $18-96$ & $18-106$ & $19-102$ \\
\hline \multicolumn{9}{|l|}{ Sex, n (\%) } \\
\hline Male & $558(54.5)$ & $506(53.1)$ & 895 (55.9) & $966(57.8)$ & $253(53.3)$ & $200(53.1)$ & $90(52.0)$ & $243(54.5)$ \\
\hline Female & $465(45.5)$ & 447 (46.9) & 707 (44.I) & $704(42.2)$ & $222(46.7)$ & I 77 (46.9) & $83(48.0)$ & $203(45.5)$ \\
\hline
\end{tabular}

Abbreviation: IQR, interquartile range.

Table 2 Clinical characteristics by province

\begin{tabular}{|c|c|c|c|c|c|c|c|c|}
\hline \multirow[t]{2}{*}{ Characteristic } & \multicolumn{2}{|l|}{ Ontario } & \multicolumn{2}{|c|}{$\begin{array}{l}\text { Alberta and British } \\
\text { Columbia }\end{array}$} & \multicolumn{2}{|c|}{ Canadian Prairie } & \multicolumn{2}{|c|}{ Atlantic Canada } \\
\hline & $\begin{array}{l}\text { Primary } \\
(\mathrm{N}=1,023)\end{array}$ & $\begin{array}{l}\text { Secondary } \\
(N=953)\end{array}$ & $\begin{array}{l}\text { Primary } \\
(\mathrm{N}=1,602)\end{array}$ & $\begin{array}{l}\text { Secondary } \\
(N=1,670)\end{array}$ & $\begin{array}{l}\text { Primary } \\
(\mathrm{N}=475)\end{array}$ & $\begin{array}{l}\text { Secondary } \\
(N=377)\end{array}$ & $\begin{array}{l}\text { Primary } \\
(\mathrm{N}=173)\end{array}$ & $\begin{array}{l}\text { Secondary } \\
(\mathrm{N}=446)\end{array}$ \\
\hline ABSSSI subtype, ${ }^{a} n(\%)$ & $\mathrm{n}=\mathrm{I}, \mathrm{I} 44$ & $\mathrm{n}=979$ & $\mathrm{n}=\mathrm{I}, 784$ & $\mathrm{n}=1,739$ & $n=518$ & $\mathrm{n}=385$ & $n=185$ & $\mathrm{n}=463$ \\
\hline Cellulitis/erysipelas & $725(63.4)$ & $568(58.0)$ & $1,121(62.8)$ & $980(56.4)$ & $289(55.8)$ & $182(47.3)$ & $114(61.6)$ & $152(32.8)$ \\
\hline Wound infection & $2(0.2)$ & $3(0.3)$ & $3(0.2)$ & $3(0.2)$ & $\mathrm{I}(0.2)$ & $\mathrm{I}(0.3)$ & 0 & $4(0.9)$ \\
\hline Abscess & $379(33.1)$ & $217(22.2)$ & $614(34.4)$ & $494(28.4)$ & $217(41.9)$ & $132(34.3)$ & $63(34.1)$ & $77(16.6)$ \\
\hline Other & $38(3.3)$ & $191(19.5)$ & $46(2.6)$ & $262(15.1)$ & II (2.I) & $70(18.2)$ & $8(4.3)$ & $230(49.7)$ \\
\hline Anatomical site of infection, ${ }^{\mathrm{b}} \mathrm{n}(\%)$ & $\mathrm{n}=991$ & $n=762$ & $\mathrm{n}=1,562$ & $\mathrm{n}=1,415$ & $n=464$ & $\mathrm{n}=307$ & $\mathrm{n}=166$ & $n=224$ \\
\hline Head/neck/face & $68(6.9)$ & $34(4.5)$ & $120(7.7)$ & $104(7.3)$ & $31(6.7)$ & $24(7.8)$ & $7(4.2)$ & $16(7.1)$ \\
\hline Limb & $712(71.8)$ & $562(73.8)$ & $1,028(65.8)$ & $946(66.9)$ & $291(62.7)$ & $217(70.7)$ & $117(70.5)$ & $142(63.4)$ \\
\hline Trunk & $165(16.6)$ & $137(18.0)$ & $337(21.6)$ & $307(21.7)$ & $124(26.7)$ & $60(19.5)$ & $36(21.7)$ & $60(26.8)$ \\
\hline Multiple & $40(4.0)$ & $20(2.6)$ & $60(3.8)$ & $27(1.9)$ & $17(3.7)$ & $5(1.6)$ & $5(3.0)$ & $3(1.3)$ \\
\hline Not determinable & $6(0.6)$ & $9(1.2)$ & $17(1.1)$ & $31(2.2)$ & $\mathrm{I}(0.2)$ & $\mathrm{I}(0.3)$ & I (0.6) & $3(1.3)$ \\
\hline Comorbid conditions, ${ }^{c} \mathrm{n}(\%)$ & $\mathrm{n}=521$ & $\mathrm{n}=1,030$ & $n=688$ & $\mathrm{n}=1,422$ & $n=219$ & $\mathrm{n}=328$ & $n=79$ & $\mathrm{n}=409$ \\
\hline Diabetes mellitus & $282(54.1)$ & $44 \mathrm{I}(42.8)$ & $354(5 \mid .5)$ & $604(42.5)$ & I57 (7I.7) & $186(56.7)$ & $57(72.2)$ & $199(48.7)$ \\
\hline Congestive heart failure & $56(10.7)$ & $163(15.8)$ & 77 (II.2) & $207(14.6)$ & $13(5.9)$ & $45(13.7)$ & $4(5.1)$ & $71(17.4)$ \\
\hline Peripheral vascular disease & $19(3.6)$ & $46(4.5)$ & $21(3.1)$ & $62(4.4)$ & $5(2.3)$ & $7(2.1)$ & $5(6.3)$ & $20(4.9)$ \\
\hline Cerebrovascular disease & II (2.I) & $29(2.8)$ & $6(0.9)$ & $56(3.9)$ & $5(2.3)$ & $12(3.7)$ & 0 & $16(3.9)$ \\
\hline Myocardial infarction & $5(1.0)$ & $4 \mid(4.0)$ & $5(0.7)$ & $56(3.9)$ & $2(0.9)$ & $8(2.4)$ & 0 & $8(2.0)$ \\
\hline Renal disease & $83(15.9)$ & $216(21.0)$ & $\mid 48(2 \mid .5)$ & $284(20.0)$ & $20(9.1)$ & $42(12.8)$ & $9(11.4)$ & $65(15.9)$ \\
\hline Malignancy & $17(3.3)$ & $59(5.7)$ & $22(3.2)$ & $84(5.9)$ & $5(2.3)$ & $15(4.6)$ & $\mathrm{I}(\mathrm{I} .3)$ & $29(7.1)$ \\
\hline Moderate or severe liver disease & $48(9.2)$ & $35(3.4)$ & $55(8.0)$ & $69(4.9)$ & $12(5.5)$ & $13(4.0)$ & $3(3.8)$ & $\mathrm{I}(0.2)$ \\
\hline \multicolumn{9}{|l|}{ Number of comorbidities, n (\%) } \\
\hline 0 & $598(58.5)$ & $295(31.0)$ & $\mathrm{I}, 054(65.8)$ & $714(42.8)$ & $286(60.2)$ & I 46 (38.7) & $105(60.7)$ & 166 (37.2) \\
\hline 1 & 345 (33.7) & $366(38.4)$ & $424(26.5)$ & $576(34.5)$ & $164(34.5)$ & I48 (39.3) & $58(33.5)$ & $170(38.1)$ \\
\hline 2 & $61(6.0)$ & 199 (20.9) & $106(6.6)$ & $270(16.2)$ & $19(4.0)$ & $62(16.4)$ & $8(4.6)$ & $82(18.4)$ \\
\hline 3 & $17(1.7)$ & $80(8.4)$ & $15(0.9)$ & $89(5.3)$ & $5(1.1)$ & $19(5.0)$ & $2(1.2)$ & $24(5.4)$ \\
\hline$>3$ & $2(0.2)$ & $13(1.4)$ & $3(0.2)$ & $21(1.3)$ & I $(0.2)$ & $2(0.5)$ & 0 & $4(0.9)$ \\
\hline
\end{tabular}

Notes: aPatients with two or more of the same subtype coded within hospitalization were counted once; patients with different subtypes coded were counted once in each category; "patients with two or more of the same site coded within hospitalization were counted only once in the category; patients with two or more site categories were counted in the "multiple" category; patients with site code and undetermined site code were counted in the "multiple category"; "site not determinable" included L02.9, L03.9, A46, L05.0, and T14.0. Patients with no site codes were not included; 'patients may have had several comorbidities; patients with two or more codes related to a single comorbidity were counted only once in the category.

Abbreviation: ABSSSI, acute bacterial skin and skin structure infections. 
Table 3 Hospitalization characteristics by province

\begin{tabular}{|c|c|c|c|c|c|c|c|c|}
\hline \multirow[t]{2}{*}{ Characteristic } & \multicolumn{2}{|l|}{ Ontario } & \multicolumn{2}{|c|}{$\begin{array}{l}\text { Alberta and British } \\
\text { Columbia }\end{array}$} & \multicolumn{2}{|c|}{ Canadian Prairie } & \multicolumn{2}{|c|}{ Atlantic Canada } \\
\hline & $\begin{array}{l}\text { Primary } \\
(\mathrm{N}=1,023)\end{array}$ & $\begin{array}{l}\text { Secondary } \\
(\mathrm{N}=953)\end{array}$ & $\begin{array}{l}\text { Primary } \\
(\mathrm{N}=1,602)\end{array}$ & $\begin{array}{l}\text { Secondary } \\
(\mathrm{N}=1,670)\end{array}$ & $\begin{array}{l}\text { Primary } \\
(\mathrm{N}=475)\end{array}$ & $\begin{array}{l}\text { Secondary } \\
(\mathrm{N}=377)\end{array}$ & $\begin{array}{l}\text { Primary } \\
(\mathrm{N}=\mid 73)\end{array}$ & $\begin{array}{l}\text { Secondary } \\
(\mathrm{N}=446)\end{array}$ \\
\hline \multicolumn{9}{|l|}{ Year hospitalized, n (\%) } \\
\hline 2008 & $104(10.2)$ & $137(14.4)$ & $240(15.0)$ & $224(13.4)$ & $36(7.6)$ & $20(5.3)$ & $25(14.5)$ & $|4|(3 \mid .6)$ \\
\hline 2009 & $128(12.5)$ & $125(13.1)$ & $205(12.8)$ & $236(14.1)$ & $54(11.4)$ & $43(11.4)$ & $26(15.0)$ & $72(16.1)$ \\
\hline 2010 & $155(15.2)$ & $145(15.2)$ & $238(14.9)$ & $247(14.8)$ & $77(16.2)$ & $60(15.9)$ & $25(14.5)$ & $74(16.6)$ \\
\hline 2011 & $152(14.9)$ & $|4|(\mid 4.8)$ & $234(14.6)$ & $249(14.9)$ & 89 (18.7) & $65(17.2)$ & $41(23.7)$ & $48(10.8)$ \\
\hline 2012 & $156(15.2)$ & $145(15.2)$ & $232(14.5)$ & $245(14.7)$ & $76(16.0)$ & $67(17.8)$ & $18(10.4)$ & $43(9.6)$ \\
\hline 2013 & $192(18.8)$ & $129(13.5)$ & $24 I(15.0)$ & $260(15.6)$ & $77(16.2)$ & $70(18.6)$ & $24(13.9)$ & $42(9.4)$ \\
\hline 2014 & $136(13.3)$ & $131(13.7)$ & $212(13.2)$ & $209(12.5)$ & $66(13.9)$ & $52(13.8)$ & $14(8.1)$ & $26(5.8)$ \\
\hline \multicolumn{9}{|l|}{ Admission type, $\mathrm{n}(\%)$} \\
\hline Emergent & $\mathrm{I}, 005(98.2)$ & $885(92.9)$ & $1,564(97.6)$ & $1,509(90.4)$ & $472(99.4)$ & $353(93.6)$ & $166(96.0)$ & $400(89.7)$ \\
\hline Elective & $18(1.8)$ & $68(7.1)$ & $38(2.4)$ & I6I (9.6) & $3(0.6)$ & $24(6.4)$ & $7(4.0)$ & $46(10.3)$ \\
\hline \multicolumn{9}{|l|}{ Hospitalization payer, n (\%) } \\
\hline Resident province & $997(97.5)$ & $944(99.1)$ & I,559 (97.3) & $\mathrm{I}, 632(97.7)$ & $460(96.8)$ & $367(97.3)$ & 159 (91.9) & $437(98.0)$ \\
\hline Other province & $18(1.8)$ & $5(0.5)$ & $31(1.9)$ & $23(1.4)$ & $14(2.9)$ & $10(2.7)$ & $8(4.6)$ & $7(1.6)$ \\
\hline Canadian resident self-pay & $6(0.6)$ & $\mathrm{I}(0.1)$ & $6(0.4)$ & $4(0.2)$ & $\mathrm{I}(0.2)$ & 0 & 0 & 0 \\
\hline Foreigner self-pay & $\mathrm{I}(0.1)$ & 0 & 0 & 0 & 0 & 0 & $\mathrm{I}(0.6)$ & 0 \\
\hline Other & $\mathrm{I}(0 . \mathrm{I})$ & $3(0.3)$ & $6(0.4)$ & II (0.7) & 0 & 0 & $5(2.9)$ & $2(0.4)$ \\
\hline
\end{tabular}

Atlantic Canada, there was an even higher ratio, over five times, of secondary vs primary hospitalizations (Table 1).

Overall, the majority of hospitalizations were related to cellulitis/erysipelas (32.8\% to $63.4 \%$ ) followed by abscess ( $16.6 \%$ to $41.9 \%$ ), and other subtypes $(2.1 \%$ to $49.7 \%$; Table 2). Cellulitis/erysipelas was the most frequent type of infection for patients with a primary diagnosis across all provinces (55.8\% to $63.4 \%$ ) and for patients with a secondary diagnosis in Ontario, Alberta/British Columbia, and the Canadian Prairie (47.3\% to $58.0 \%$ ). In all provinces, infections categorized as "other" occurred more frequently among patients with a secondary diagnosis than among those with a primary diagnosis. In the total cohort, the majority of infections involved a limb $(62.7 \%$ to $73.8 \%)$, followed by the trunk (16.6\% to $26.8 \%$ ). The most common comorbid conditions were diabetes mellitus $(42.5 \%$ to $72.2 \%)$, renal disease $(9.1 \%$ to $21.5 \%)$, and congestive heart failure $(5.1 \%$ to $17.4 \%$ ). The distribution of comorbidity burden differed slightly depending on whether the patient had a primary or secondary diagnosis. The majority of the patients across the samples had zero or one comorbidity; however, among patients with a secondary diagnosis, a higher proportion of patients had two or more comorbidities.

The number and percentage of patients with a primary or secondary diagnosis appeared to be relatively consistent from 2008 to 2014; the only exception was Atlantic Canada, where the proportion of patients with a secondary diagnosis decreased from $31.6 \%$ in 2008 to $5.8 \%$ in 2014 (Table 3).
The majority of hospitalizations were emergent $(96.0 \%$ to $99.4 \%$ for patients with a primary diagnosis and $89.7 \%$ to $93.6 \%$ for patients with a secondary diagnosis) (Table 3 ). Of the 365 patients with elective hospital admissions, a majority had a secondary diagnosis ranging from $79.1 \%$ in Ontario to $88.9 \%$ in Canadian Prairie. More than $90 \%$ of the hospitalization costs were paid by the resident province (Table 3).

\section{Hospitalization outcomes}

Hospitalization outcomes for the entire study period (20082014) are shown in Table 4 and for the most recent year (2014) are shown in Table 5. The mean LOS over the study period varied according to the geographic region, from 9 to 18 days for primary diagnosis and from 25 to 43 days for secondary diagnosis (Table 4). In the final year of the study (2014), LOS ranged from 7.7 to 13.4 days for a primary diagnosis and from 18.2 to 25.2 days for a secondary diagnosis. LOS in a specialty care facility varied by geographical region, ranging from 83 to 157 hours for patients with a primary diagnosis and from 153 to 203 hours for patients with a secondary diagnosis.

Over the entire study period, the majority of hospitalized patients were discharged to their residence; rates of discharge to home were higher among patients with a primary diagnosis compared with patients with a secondary diagnosis $(77.7 \%$ to $82.7 \%$ vs $56.8 \%$ to $64.5 \%$, respectively). ABSSSI due to MRSA as a secondary diagnosis was associated with higher in-hospital mortality rates $(7.2 \%$ to $12.1 \%)$ than 
Table 4 Outcomes (all data from 2008-20I4) by province

\begin{tabular}{|c|c|c|c|c|c|c|c|c|}
\hline \multirow[t]{2}{*}{ Characteristic } & \multicolumn{2}{|l|}{ Ontario } & \multicolumn{2}{|c|}{$\begin{array}{l}\text { Alberta and British } \\
\text { Columbia }\end{array}$} & \multicolumn{2}{|c|}{ Canadian Prairie } & \multicolumn{2}{|c|}{ Atlantic Canada } \\
\hline & $\begin{array}{l}\text { Primary } \\
(\mathrm{N}=1,023)\end{array}$ & $\begin{array}{l}\text { Secondary } \\
(\mathrm{N}=953)\end{array}$ & $\begin{array}{l}\text { Primary } \\
(\mathrm{N}=1,602)\end{array}$ & $\begin{array}{l}\text { Secondary } \\
(\mathrm{N}=1,670)\end{array}$ & $\begin{array}{l}\text { Primary } \\
(N=475)\end{array}$ & $\begin{array}{l}\text { Secondary } \\
(\mathrm{N}=377)\end{array}$ & $\begin{array}{l}\text { Primary } \\
(\mathrm{N}=173)\end{array}$ & $\begin{array}{l}\text { Secondary } \\
(\mathrm{N}=446)\end{array}$ \\
\hline \multicolumn{9}{|l|}{ Hospital LOS, days } \\
\hline Mean & 9 & 25 & 13 & 33 & 12 & 31 & 18 & 43 \\
\hline Standard deviation & 12 & 41 & 21 & 61 & 25 & 54 & 42 & 92 \\
\hline Median & 6 & 13 & 7 & 16 & 6 & 12 & 7 & 18 \\
\hline IQR & $3-10$ & $7-27$ & $4-14$ & $7-37$ & $3-11$ & $6-30$ & $4-14$ & $7-44$ \\
\hline Range & $1-157$ & $1-662$ & $|-4| 2$ & $1-1,167$ & $1-259$ & $\mathrm{I}-553$ & $1-349$ & $|-|, 3 \mid 3$ \\
\hline Special care facility LOS, hours & $\mathrm{n}=37$ & $n=173$ & $n=33$ & $n=170$ & $n=8$ & $n=62$ & $n=9$ & $n=170$ \\
\hline Mean & 137 & 200 & 83 & 172 & 157 & 153 & 111 & 203 \\
\hline Standard deviation & 129 & 399 & 67 & 193 & 291 & 163 & 135 & 283 \\
\hline Median & 87 & 113 & 57 & 114 & 39 & 107 & 61 & 120 \\
\hline IQR & $43-216$ & $49-189$ & $32-135$ & $53-205$ & $21-130$ & $62-166$ & $24-95$ & $54-258$ \\
\hline Range & $4-546$ & $4-4,343$ & $3-234$ & $5-1,435$ & $18-858$ & $17-964$ & $17-408$ & $5-1,447$ \\
\hline \multicolumn{9}{|l|}{ Main patient service, $\mathrm{n}(\%)$} \\
\hline General medicine & $74 I(72.4)$ & $555(58.2)$ & $\mathrm{I}, 237(77.2)$ & $952(57.0)$ & $324(68.2)$ & $232(6 \mid .5)$ & $58(33.5)$ & $132(29.6)$ \\
\hline Dermatology & $52(5.1)$ & $5(0.5)$ & $45(2.8)$ & $7(0.4)$ & 0 & 0 & 0 & 0 \\
\hline General surgery & $91(8.9)$ & $75(7.9)$ & $178(|I|)$. & $232(13.9)$ & $79(16.6)$ & $30(8.0)$ & $33(19.1)$ & $56(12.6)$ \\
\hline Other surgery & 79 (7.7) & $112(11.8)$ & $48(3.0)$ & III (6.6) & $26(5.5)$ & $26(6.9)$ & $26(15.0)$ & $66(14.8)$ \\
\hline Infectious diseases & $3(0.3)$ & $4(0.4)$ & 0 & 0 & 0 & 0 & $2(1.2)$ & $5(1.1)$ \\
\hline Other & $57(5.6)$ & $202(2 \mid .2)$ & $94(5.9)$ & $368(22.0)$ & $46(9.7)$ & $89(23.6)$ & $54(31.2)$ & $187(41.9)$ \\
\hline \multicolumn{9}{|l|}{ Discharge status, n (\%) } \\
\hline Died in hospital & $16(1.6)$ & $89(9.3)$ & $25(1.6)$ & 140 (8.4) & $2(0.4)$ & $27(7.2)$ & $\mathrm{I}(0.6)$ & $54(12.1)$ \\
\hline Discharged to home & $811(79.3)$ & $54 \mid(56.8)$ & $\mathrm{I}, 244(77.7)$ & I,067 (63.9) & $392(82.5)$ & $243(64.5)$ & $143(82.7)$ & $268(60.1)$ \\
\hline Continuing care & $124(12.1)$ & 231 (24.2) & $106(6.6)$ & $183(11.0)$ & $22(4.6)$ & $40(10.6)$ & $14(8.1)$ & $70(15.7)$ \\
\hline Other & $72(7.0)$ & $92(9.7)$ & $227(14.2)$ & $280(16.8)$ & $59(12.4)$ & $67(17.8)$ & $15(8.7)$ & $54(12.1)$ \\
\hline \multicolumn{9}{|l|}{ Surgical procedures, n (\%) } \\
\hline Incision & $207(3 \mathrm{I} . \mathrm{I})$ & $98(19.0)$ & $237(26.9)$ & $152(14.8)$ & $97(36.9)$ & $52(2 \mid .4)$ & $36(32.7)$ & 25 (II.I) \\
\hline Drainage & $254(38.1)$ & $129(25.0)$ & $306(34.7)$ & $202(19.6)$ & II4 (43.3) & $61(25.1)$ & $38(34.5)$ & $35(15.6)$ \\
\hline Surgical debridement & $128(19.2)$ & $136(26.4)$ & $205(23.3)$ & $391(38.0)$ & $40(15.2)$ & $71(29.2)$ & $22(20.0)$ & $101(44.9)$ \\
\hline Excision of wound & $17(2.6)$ & $25(4.9)$ & $43(4.9)$ & $50(4.9)$ & $6(2.3)$ & $8(3.3)$ & $4(3.6)$ & $7(3.1)$ \\
\hline Amputation & $60(9.0)$ & $127(24.7)$ & $90(10.2)$ & $235(22.8)$ & $6(2.3)$ & $51(21.0)$ & $10(9.1)$ & $57(25.3)$ \\
\hline
\end{tabular}

Abbreviations: IQR, interquartile range; LOS, length of stay.

Table 5 Outcomes in 2014 by province

\begin{tabular}{|c|c|c|c|c|c|c|c|c|}
\hline \multirow[t]{2}{*}{ Characteristic } & \multicolumn{2}{|l|}{ Ontario } & \multicolumn{2}{|c|}{$\begin{array}{l}\text { Alberta and British } \\
\text { Columbia }\end{array}$} & \multicolumn{2}{|c|}{ Canadian Prairie } & \multicolumn{2}{|c|}{ Atlantic Canada } \\
\hline & $\begin{array}{l}\text { Primary } \\
\text { diagnosis }\end{array}$ & $\begin{array}{l}\text { Secondary } \\
\text { diagnosis }\end{array}$ & $\begin{array}{l}\text { Primary } \\
\text { diagnosis }\end{array}$ & $\begin{array}{l}\text { Secondary } \\
\text { diagnosis }\end{array}$ & $\begin{array}{l}\text { Primary } \\
\text { diagnosis }\end{array}$ & $\begin{array}{l}\text { Secondary } \\
\text { diagnosis }\end{array}$ & $\begin{array}{l}\text { Primary } \\
\text { diagnosis }\end{array}$ & $\begin{array}{l}\text { Secondary } \\
\text { diagnosis }\end{array}$ \\
\hline Hospital LOS, days & $\mathrm{n}=136$ & $n=136$ & $\mathrm{n}=212$ & $\mathrm{n}=209$ & $n=66$ & $\mathrm{n}=52$ & $\mathrm{n}=14$ & $n=26$ \\
\hline Mean & 7.7 & 18.2 & 12.4 & 25.0 & 13.4 & 18.3 & 8.9 & 25.2 \\
\hline Standard deviation & 7.0 & 21.3 & I5.I & 32.1 & 21.8 & 18.1 & 10.8 & 35.6 \\
\hline Special care facility LOS, hours & $\mathrm{n}=2$ & $\mathrm{n}=27$ & $n=5$ & $n=21$ & $\mathrm{n}=2$ & $n=6$ & $\mathrm{n}=0$ & $n=6$ \\
\hline Mean & 161 & 160 & 57 & 142 & 439 & 92 & - & 170 \\
\hline Standard deviation & 78.5 & 150.0 & 44.7 & 190.1 & 593.3 & 47.6 & - & 235.4 \\
\hline \multicolumn{9}{|l|}{ Main patient service, $\mathrm{n}(\%)$} \\
\hline General medicine & II 5 (84.6) & $76(58.0)$ & 168 (79.2) & $126(60.3)$ & $45(68.2)$ & $39(75.0)$ & $4(28.6)$ & $6(23.1)$ \\
\hline Dermatology & $6(4.4)$ & I (0.8) & $8(3.8)$ & 0 & 0 & 0 & 0 & 0 \\
\hline General surgery & $5(3.7)$ & $10(7.6)$ & $23(10.8)$ & $22(10.5)$ & $9(13.6)$ & $5(9.6)$ & $2(14.3)$ & $6(23.1)$ \\
\hline Other surgery & $4(2.9)$ & $16(12.2)$ & $4(1.9)$ & $13(6.2)$ & $4(6.1)$ & $2(3.8)$ & $5(35.7)$ & 5 (19.2) \\
\hline Infectious diseases & 0 & 0 & 0 & $48(23.0)$ & 0 & 0 & I (7.I) & 0 \\
\hline Other & $6(4.4)$ & $28(2 \mid .4)$ & $9(4.2)$ & 0 & $8(12.1)$ & $6(11.5)$ & $2(14.3)$ & $9(34.6)$ \\
\hline \multicolumn{9}{|l|}{ Surgical procedures, n (\%) } \\
\hline Incision & $28(34.1)$ & $17(22.1)$ & $28(25.9)$ & $16(14.4)$ & $14(42.4)$ & $10(2 \mathrm{I} .7)$ & I (20) & $2(10.5)$ \\
\hline Drainage & $31(37.8)$ & $28(36.4)$ & $34(31.5)$ & $21(18.9)$ & $14(42.4)$ & I 3 (28.3) & I (20) & $5(26.3)$ \\
\hline Surgical debridement & $15(18.3)$ & II (I4.3) & $24(22.2)$ & $40(36.0)$ & $3(9.1)$ & $16(34.8)$ & I (20) & $6(3 \mid .6)$ \\
\hline Excision of wound & I (I.2) & $6(7.8)$ & $10(9.3)$ & $8(7.2)$ & I (3.0) & I (2.2) & I (20) & $3(15.8)$ \\
\hline Amputation & $7(8.5)$ & $15(19.5)$ & $12(I I . I)$ & $26(23.4)$ & I (3.0) & $6(13.0)$ & I (20) & $3(15.8)$ \\
\hline
\end{tabular}

Abbreviation: LOS, length of stay. 
infections identified as a primary diagnosis $(0.4 \%$ to $1.6 \%)$. The proportion of patients receiving continuing care was also higher among patients with a secondary diagnosis $(10.6 \%$ to $24.2 \%)$ than those with a primary diagnosis (4.6\% to $12.1 \%$; Table 4$)$.

The most common procedures overall and over the study period were drainage $(15.6 \%$ to $43.3 \%)$, followed by surgical debridement ( $15.2 \%$ to $44.9 \%)$, incision $(11.1 \%$ to $36.9 \%$ ), amputation (2.3\% to $25.3 \%$ ), and excision of wound $(2.3 \%$ to $4.9 \%)$. Drainage was the most common surgical procedure in primary diagnosis patients in 2014 (20\% to $42.4 \%)$ and over the study period (34.5\% to $43.3 \%)$, whereas among patients with a secondary diagnosis, surgical debridement was most common across all provinces $(26.4 \%$ to $44.9 \%)$ over the study period and in all provinces other than Ontario in 2014. Amputations were more common among patients with ABSSSI due to MRSA as a secondary diagnosis (21.0\% to $25.3 \%$ ), than patients with a primary diagnosis $(2.3 \%$ to $10.2 \%)$.

\section{Time trends: 2008-20/4}

In patients with a primary diagnosis of ABSSSI due to MRSA and who were hospitalized in either Ontario or Atlantic Canada, there was a trend toward decreasing overall mean hospital LOS from 2008 (11.5 days in Ontario, 14.0 days in Atlantic Canada) to 2014 (7.7 days in Ontario, 8.9 days in Atlantic Canada), with some variability in the intermittent years (Tables S2 and S3). Mean hospital LOS in the Alberta/ British Columbia and Canadian Prairie regions was more consistent from 2008 to 2014, with variability observed in the interim period (Tables S4 and S5).

In patients with a secondary diagnosis across all regions, there was a trend of decreasing mean hospital LOS from 2008 to 2014 , with variability observed in the intermittent years. There was a decrease of 13 days in Ontario (from 31.5 to 18.2 days; Table S2), 12 days in Alberta/British Columbia (from 37.1 to 25.0 days; Table S4), 30 days in Canadian Prairie (from 48.5 to 18.3 days; Table S5), and 22 days in Atlantic Canada (from 47.6 to 25.2 days; Table S3).

\section{Discussion}

This is the first study to examine hospital LOS and time trends of hospital LOS, using data as recent as 2014, for ABSSSI due to MRSA across multiple geographic regions in Canada, excluding Quebec. In addition, this study is the first to use an algorithm to distinguish patients based on their primary reason for hospitalization and quantify results based on whether patients were hospitalized primarily for ABSSSI due to MRSA or another condition.
The majority of patients with a primary diagnosis of ABSSSI caused by MRSA were younger (18 to 44 years) and had fewer comorbid conditions than those with a secondary diagnosis. However, patients aged 65 years or older across all provinces were more likely to be hospitalized with ABSSSI due to MRSA as a secondary diagnosis compared with a primary diagnosis. This could imply that those in the secondary diagnosis group might have acquired the infection during hospitalization or that other conditions might have motivated the admission, but ABSSSI due to MRSA was also present at the time of admission. Because medical chart data were not available to detail the sequence of events, this cannot be confirmed using the DAD database. Regardless of the exact scenarios, hospital LOS for this patient group was likely influenced by comorbidities in addition to the ABSSSI caused by MRSA diagnosis itself. However, we did observe that those with a primary diagnosis generally had a shorter LOS, lower mortality and need for continued care, higher discharge rates, and higher rates of discharge to home than patients with a secondary diagnosis.

Analysis across the regions showed that the most common infection types were cellulitis followed by abscess. Common treatments vary by infection type and some may require more hospital resources than others. For example, an abscess requires incision and drainage, whereas cellulitis/erysipelas might require emergent surgical debridement in cases of severe infections in which a necrotizing process is suspected. ${ }^{33}$ These procedures were observed in this study, with incision and drainage occurring more frequently in patients with a primary infection and surgical debridement occurring more frequently in patients with a secondary infection. Additional research regarding the association between infection type, specific resource utilization, and related micro-costing could be useful in evaluating the total cost of care.

Baibergenova et al evaluated factors associated with prolonged LOS in patients hospitalized for cellulitis as the primary diagnosis, defining "prolonged LOS" as greater than 7 days. $^{32}$ As seen in this analysis, the LOS for the majority of the patients can be considered "prolonged". Using a multivariate analysis, Baibergenova et al identified factors associated with prolonged admission as female sex; patient age, where patients older than 65 years were at increased risk for longer LOS and patients older than 75 years incurred an even greater risk; congestive heart failure as a comorbidity; dermatology consultation; and admission to or consultation by a surgical service. Dermatology consultation was associated with the highest odds of longer LOS (odds ratio: 4.5). Although not explicitly examined, it is interesting to consider 
how these factors may contribute to the longer LOS observed in our study.

Although the overall observation was that cellulitis and abscess comprised the majority of the infections for both primary and secondary diagnoses, patients with a secondary diagnosis in the Atlantic Canada region have a notably different demographic patterns with these infection types; cellulitis and abscess made up just less than half of all infections, and nearly half of all infections were classified as "other". In addition, over the study period, this region reported more than double the number of secondary diagnosis than primary diagnosis, whereas other regions had comparable numbers of patients with primary and secondary infections. Since $\sim 60 \%$ of the patients with secondary infection were 65 years or older in the Atlantic Canada province, this might be a contributing factor to the higher numbers of secondary infections reported. Additional research is needed to fully elucidate the factors that might contribute to these findings.

The data in this study show good consistency with data from another Canadian study that reported a mean LOS of 7.1 days and 16.1 days for patients admitted for cellulitis as a primary condition and as a comorbidity, respectively. ${ }^{32}$ However, a key limitation of that study is the unknown proportion of patients with an MRSA infection in the cohort. The mean hospital LOS observed in this study is notably longer than that reported for admissions due to all causes in Canadian hospitals. The Organization for Economic Cooperation and Development reported that the average Canadian LOS for patients admitted for all causes was 7.6 to 7.7 days during the years 2008-2012. ${ }^{34}$ An evaluation by the CIHI reported the average LOS as 7.2 days for all of Canada, with an average LOS of 6.7 days for Canada excluding Quebec from 2004 to $2005 .{ }^{35}$ In addition, although the mean LOS reported herein ranged from 9 to 18 days for primary diagnosis and from 25 to 43 days for secondary diagnosis over the study period, the high end of the ranges and analysis of the 75 th percentile of the interquartile range indicate substantial outliers at the higher end of the LOS spectrum.

The Canadian LOS data described in this study are similar to data reported in population-wide studies in the USA. LOS data obtained between 2002 and 2006 for cSSTI due to MRSA showed a mean of 12.6 (SD: 18.9) days and a median LOS of 7.0 days. ${ }^{21}$ For SSTI caused by $S$. aureus, mean hospital LOS based on information in the HCUP database was 7.3 days in 2009 for hospitalized adult and pediatric patients; LOS decreased from 9.9 days in $2001 .^{7}$ Similarly, in another study that evaluated hospitalization outcomes using the Premier Perspective database, the in-hospital mean and median LOS were 6.1 and 5 days, respectively, for a diagnosis of SSSI due to $S$. aureus. ${ }^{30}$ A key limitation to these studies is the unknown proportion of patients with an MRSA infection in the cohort.

Compared with European hospital LOS data for cSSTI caused by MRSA as a primary diagnosis, representing a 1-year period between July 2010 and June 2011, the mean LOS in Canada in 2014 was lower (7.7 to 13.4 days); the mean LOS in Europe was 20.6 days with a range from 15.2 days in the UK to 25.0 days in Portugal. ${ }^{28}$ This is consistent with clinical trial data that have shown a longer LOS for cSSTI caused by MRSA in Europe than in other world regions. ${ }^{36}$

A secondary diagnosis of ABSSSI due to MRSA generally entailed a longer LOS in both the hospital and the specialty care facility, with some exceptions. The longer LOS for patients with a secondary diagnosis is not unexpected because these patients were generally older and represented a sicker population, as determined by the higher comorbidity burden. In general, comorbidities might predict a longer LOS because of the more involved clinical management required to treat both the patient's infection and other conditions. Patients with secondary diagnoses also underwent more complex surgical procedures (surgical debridement and amputation), which might have contributed to longer hospital stays. Consistent with what was observed in this analysis, other studies have linked longer hospital stays and increased mortality rate in hospitalized patients with skin infections to older age and comorbidities. $^{23,30,37}$

In 2014, the most recent year of the study, the interprovincial differences in LOS between primary and secondary diagnosis demonstrated notable variability, from 7.7 days in Ontario to 13.4 days in Canadian Prairie for primary diagnosis and 18.2 days in Ontario to 25.2 days in Atlantic Canada for secondary diagnosis. Intraprovincial LOS variations in primary and secondary diagnosis were noted as well, with 1.5-fold to threefold differences in LOS noted for patients with a secondary diagnosis compared with patients with a primary diagnosis.

Based on an analysis of the special care facility data, two potential populations can be proposed from this data set: those who require hospitalization only and those who require further medical service at discharge, with medical care extended through the use of a special care facility. Although a minority of patients required special care facility services, those who used the service required a mean of 10 to 25 days of additional care. A higher portion of patients with secondary infections required use of a special care facility, with the proportions of patients ranging from $1.7 \%$ to $5.2 \%$ 
for primary infections and $10.2 \%$ to $38.1 \%$ for secondary infections. This analysis did not investigate the demographics and potential factors associated with special care facility use of this resource-intense subgroup, but this could be the subject of future research.

Although the time trends showed variability over the years and lack of a linear decline, a general trend toward shorter mean LOS for patients with a secondary diagnosis between 2008 and 2014 was observed across all geographic regions; the Canadian Prairie had the greatest reduction in LOS (a reduction of 30 days from 48.5 days in 2008 to 18.3 days in 2014). The time trend for primary diagnosis was less consistently defined across the regions, with variability over the years. However, a trend toward decreasing hospital LOS between 2008 and 2014 was observed in Ontario and Atlantic Canada; the Alberta/British Columbia and Canadian Prairie provinces had consistent hospital LOS. Decreases in LOS may be due to the increased use of antibiotics in the outpatient setting, either due to the availability of oral medications or the implementation of outpatient-setting IV infusion centers or home-infusion capabilities.

Hospitals are the largest category of national health expenditure in Canada. With hospitalization costs averaging US\$1,000/day, ${ }^{38-40}$ the cost of managing an episode of primary ABSSSI due to MRSA can average more than US\$7,000/stay. The observed longer LOS for patients diagnosed with a secondary infection, coupled with greater comorbid conditions and complex surgical procedures, might result in a higher average cost of secondary diagnosis compared with primary diagnosis. Given the lower LOS and need for ancillary care and greater discharge rate, costs associated with primary ABSSSI caused by MRSA diagnoses are minimized; further cost reductions can be achieved by shifting care from an inpatient to an outpatient setting.

\section{Limitations}

This study had several limitations. In creating the operational definition of ABSSSI, the accuracy of using diagnosis codes that appeared, on acute inpatient records, to identify a population of patients with ABSSSI due to MRSA is not known. The accuracy of diagnosis codes to identify conditions varied depending on the type of diagnosis, and identifying the severity of infections in the context of patients who have underlying comorbidities is challenging. Therefore, the study was likely subject to some misclassification bias as a result of coding errors or inclusion of codes to rule out specific diagnoses, such as diabetic foot infections. In addition, this study did not control for infection severity or distinguish whether the infection was widespread or localized. The evolving definitions of skin infection made collection of historical real-world data more challenging. Because diabetic foot infection does not have a specific ICD-10-CA code (codes E10.70 and E10.71 refer to diabetes with foot ulcer and diabetes with foot ulcer with gangrene, respectively, but do not specify whether infection is present), it was not possible to exclude this code; hence, some patients with diabetic foot infection might have been included in our sample. The general ICD-10-CA code for diabetic foot (E10.7 for diabetes with complications) was not used as an exclusion criterion because we did not want to eliminate a proportion of patients. Nevertheless, we believe that this study population is representative of the ABSSSI due to MRSA population because the selected ICD-10-CA diagnosis codes were consistent with those used in other ABSSSI database studies and, most important, with codes that fall under the new FDA-ABSSSI definition.

A further limitation was that it was not possible to examine hospitalization outcomes according to the specific antibiotic used because information related to the use of specific prescription medications and health services during the hospital stay was not available in the DAD. Since the study focused on outcomes experienced within the hospitalization episode, the results are limited by lack of potentially relevant patient information prior to hospitalization and after discharge. Not all Canadian provinces were included in the analysis because data for Quebec were not available. In addition, all regions did not contribute data equally; Atlantic Canada and the Canadian Prairie together contributed $<25 \%$ of patients, although these low numbers may reflect the actual burden of ABSSSI due to MRSA.

\section{Conclusion}

In conclusion, this study is the first to provide current realworld data on hospitalization patterns across Canada for patients with a primary or secondary diagnosis of ABSSSI due to MRSA. The mean LOS for hospitalized Canadian patients ranged from 7.7 to 13.4 days across the geographic regions, which was shorter than that reported for Europe but similar to that in the USA. Some differences in practice patterns were noted across geographic regions. These data can be used to inform best practices to optimize care with strategies such as medication management, which may facilitate hospital discharge or hospital avoidance altogether.

\section{Acknowledgments}

Parts of this material are based on data and information provided by the CIHI. However, the analyses, conclusions, 
opinions, and statements expressed herein are those of the authors and not necessarily those of the CIHI. Funding for this research was provided by Merck \& Co, Inc., Kenilworth, NJ, USA. Dominik Wolf, MSc, an employee of Merck \& Co, Inc., Kenilworth, NJ, USA, critically reviewed the manuscript. Medical writing and editorial assistance was provided by Norma I Padilla, $\mathrm{PhD}$, and Tracy T Cao, $\mathrm{PhD}$, of ApotheCom, Yardley, PA, USA. This assistance was funded by Merck \& Co, Inc., Kenilworth, NJ, USA.

\section{Author contributions}

All authors are responsible for the work described in this paper. All authors were involved in at least one of the following: conception, design, acquisition, analysis, statistical analysis, or interpretation of data. All authors were involved in drafting or revising the manuscript for important intellectual content. All authors provided final approval of the version to be submitted.

\section{Disclosure}

MHP, RL, and LH were employees of Merck Sharp \& Dohme Corp, a subsidiary of Merck \& Co, Inc., Kenilworth, NJ, USA, at the time the study was conducted and may potentially own stock and/or hold stock options in the Company. MS and JL are employees of Evidera, which received consulting fees from Merck \& Co, Inc., Kenilworth, NJ, USA. The authors report no other conflicts of interest in this work.

\section{References}

1. US Department of Health and Human Services. Center for Drug Evaluation and Research (CDER). Guidance for industry: acute bacterial skin and skin structure infections: developing drugs for treatment. US Department of Health and Human Services website. Available from: http://www.fda.gov/ downloads/Drugs/Guidances/ucm071185.pdf 2013. Accessed June 12, 2015.

2. Moet GJ, Jones RN, Biedenbach DJ, Stilwell MG, Fritsche TR. Contemporary causes of skin and soft tissue infections in North America, Latin America, and Europe: report from the SENTRY Antimicrobial Surveillance Program (1998-2004). Diagn Microbiol Infect Dis. 2007;57: $7-13$.

3. Talan DA, Krishnadasan A, Gorwitz RJ, et al; Comparison of Staphylococcus aureus from skin and soft-tissue infections in US emergency department patients, 2004 and 2008. Clin Infect Dis. 2011;53: 144-149.

4. Meddles-Torres C, Hu S, Jurgens C. Changes in prescriptive practices in skin and soft tissue infections associated with the increased occurrence of community acquired methicillin resistant Staphylococcus aureus. J Infect Public Health. 2013;6:423-430.

5. Sader HS, Farrell DJ, Jones RN. Antimicrobial susceptibility of Grampositive cocci isolated from skin and skin-structure infections in European medical centres. Int J Antimicrob Agents. 2010;36:28-32.

6. Edelsberg J, Taneja C, Zervos M, et al; Trends in US hospital admissions for skin and soft tissue infections. Emerg Infect Dis. 2009;15: $1516-1518$.
7. Suaya JA, Mera RM, Cassidy A, et al; Incidence and cost of hospitalizations associated with Staphylococcus aureus skin and soft tissue infections in the United States from 2001 through 2009. BMC Infect Dis. 2014;14:296.

8. Murray H. MRSA - happy and thriving in Canada. What's an emergency doctor supposed to do? CJEM. 2009;11:417-422.

9. Marra F, Patrick DM, Chong M, McKay R, Hoang L, Bowie WR. Population-based study of the increased incidence of skin and soft tissue infections and associated antimicrobial use. Antimicrob Agents Chemother. 2012;56:6243-6249.

10. Adam HJ, Allen VG, Currie A, et al; Community-associated methicillinresistant Staphylococcus aureus: prevalence in skin and soft tissue infections at emergency departments in the Greater Toronto Area and associated risk factors. CJEM. 2009;11:439-446.

11. Stenstrom R, Grafstein E, Romney M, et al; Prevalence of and risk factors for methicillin-resistant Staphylococcus aureus skin and soft tissue infection in a Canadian emergency department. CJEM. 2009;11: 430-438.

12. Borgundvaag B, Ng W, Rowe B, Katz K. Prevalence of methicillinresistant Staphylococcus aureus in skin and soft tissue infections in patients presenting to Canadian emergency departments. CJEM. 2013; 15:141-160.

13. Simor AE, Gilbert NL, Gravel D, et al; Methicillin-resistant Staphylococcus aureus colonization or infection in Canada: National Surveillance and Changing Epidemiology, 1995-2007. Infect Control Hosp Epidemiol. 2010;31(4):348-356.

14. Rennie RP, Jones RN, Mutnick AH. Occurrence and antimicrobial susceptibility patterns of pathogens isolated from skin and soft tissue infections: report from the SENTRY Antimicrobial Surveillance Program (United States and Canada, 2000). Diagn Microbiol Infect Dis. 2003;45:287-293.

15. Edelsberg J, Berger A, Weber DJ, Mallick R, Kuznik A, Oster G. Clinical and economic consequences of failure of initial antibiotic therapy for hospitalized patients with complicated skin and skin-structure infections. Infect Control Hosp Epidemiol. 2008;29:160-169.

16. Davis SL, Perri MB, Donabedian SM, et al; Epidemiology and outcomes of community-associated methicillin-resistant Staphylococcus aureus infection. J Clin Microbiol. 2007;45:1705-1711.

17. Labreche MJ, Lee GC, Attridge RT, et al; Treatment failure and costs in patients with methicillin-resistant Staphylococcus aureus (MRSA) skin and soft tissue infections: a South Texas Ambulatory Research Network (STARNet) study. J Am Board Fam Pract. 2013;26:508-517.

18. Sreeramoju P, Porbandarwalla NS, Arango J, et al; Recurrent skin and soft tissue infections due to methicillin-resistant Staphylococcus aureus requiring operative debridement. Am J Surg. 2011;201:216-220.

19. Tattevin P, Schwartz BS, Graber CJ, et al; Concurrent epidemics of skin and soft tissue infection and bloodstream infection due to communityassociated methicillin-resistant Staphylococcus aureus. Clin Infect Dis. 2012;55:781-788.

20. Engemann JJ, Carmeli Y, Cosgrove SE, et al; Adverse clinical and economic outcomes attributable to methicillin resistance among patients with Staphylococcus aureus surgical site infection. Clin Infect Dis. 2003;36:592-598.

21. Itani KM, Merchant S, Lin SJ, Akhras K, Alandete JC, Hatoum HT. Outcomes and management costs in patients hospitalized for skin and skin-structure infections. Am J Infect Control. 2011;39:42-49.

22. Lipsky BA, Weigelt JA, Gupta V, Killian A, Peng MM. Skin, soft tissue, bone, and joint infections in hospitalized patients: epidemiology and microbiological, clinical, and economic outcomes. Infect Control Hosp Epidemiol. 2007;28:1290-1298.

23. Ostermann H, Blasi F, Medina J, Pascual E, McBride K, Garau J. Resource use in patients hospitalized with complicated skin and soft tissue infections in Europe and analysis of vulnerable groups: the REACH study. J Med Econ. 2014;17:719-729.

24. Goetghebeur M, Landry PA, Han D, Vicente C. Methicillin-resistant Staphylococcus aureus: a public health issue with economic consequences. Can J Infect Dis Med Microbiol. 2007;18:27-34. 
25. Nathwani D. Impact of methicillin-resistant Staphylococcus aureus infections on key health economic outcomes: does reducing the length of hospital stay matter? J Antimicrob Chemother. 2003;51 Suppl 2:ii37-ii44.

26. Stephens JM, Gao X, Patel DA, Verheggen BG, Shelbaya A, Haider S. Economic burden of inpatient and outpatient antibiotic treatment for methicillin-resistant Staphylococcus aureus complicated skin and soft-tissue infections: a comparison of linezolid, vancomycin, and daptomycin. Clinicoecon Outcomes Res. 2013;5:447-457.

27. Pettigrew M, Thirion DJ, Libman M, Zanotti G. Cost comparison of linezolid versus vancomycin for treatment of complicated skin and skin-structure infection caused by methicillin-resistant Staphylococcus aureus in Quebec. Can J Infect Dis Med Microbiol. 2012;23: $187-195$.

28. Eckmann C, Lawson W, Nathwani D, et al; Antibiotic treatment patterns across Europe in patients with complicated skin and soft-tissue infections due to meticillin-resistant Staphylococcus aureus: a plea for implementation of early switch and early discharge criteria. Int $J$ Antimicrob Agents. 2014;44:56-64.

29. McKinnon PS, Goff DA, Boening AJ, Lodise TA. Health outcomes of clinically relevant patient subpopulations treated with daptomycin for methicillin-resisitant Staphylococcus aureus (MRSA) skin infection. Value Health. 2011;14:A114.

30. Menzin J, Marton JP, Meyers JL, Carson RT, Rothermel CD, Friedman M. Inpatient treatment patterns, outcomes, and costs of skin and skin structure infections because of Staphylococcus aureus. Am J Infect Control. 2010;38:44-49.

31. Conly JM, Stiver HG, Weiss KA, Becker DL, Rosner AJ, Miller E. A retrospective analysis of practice patterns in the treatment of methicillin-resistant Staphylococcus aureus skin and soft tissue infections at three Canadian tertiary care centres. Can J Infect Dis Med Microbiol. 2003;14:315-321.

32. Baibergenova A, Drucker AM, Shear NH. Hospitalizations for cellulitis in Canada: a database study. J Cutan Med Surg. 2014;18:33-37.
33. Stevens DL, Bisno AL, Chambers HF, et al; Practice guidelines for the diagnosis and management of skin and soft tissue infections: 2014 update by the Infectious Diseases Society of America. Clin Infect Dis. 2014;59:e10-e52.

34. OECD(2015), Length of hospital stay (indicator). doi: 10.1787/8dda6b7a-en. Available from: https://data.oecd.org/healthcare/length-of-hospital-stay. htm. Accessed October 18, 2015.

35. Canadian Institute for Health Information. Inpatient hospitalizations and average length of stay trends in Canada, 2003-2004 and 2004-2005. Available from: https://secure.cihi.ca/free_products/ hmdb_analysis_in_brief_e.pdf. Accessed October 18, 2015.

36. Itani KM, Sorensen S, Stokes M, Shelbaya P, McKinnon P. A regional comparison of resource utilization in patients with methicillin-resistant Staphylococcus aureus (MRSA) complicated skin and soft tissue infections (cSSTI) treated with linezolid vs vancomycin. Paper presented at: 49th Interscience Conference on Antimicrobial Agents and Chemotherapy (ICAAC); 2009; Washington, DC. Abstract O-1791.

37. Garau J, Ostermann H, Medina J, Avila M, McBride K, Blasi F. Current management of patients hospitalized with complicated skin and soft tissue infections across Europe (2010-2011): assessment of clinical practice patterns and real-life effectiveness of antibiotics from the REACH study. Clin Microbiol Infect. 2013;19:E377-E385.

38. Miller MA, Hyland M, Ofner-Agostini M, Gourdeau M, Ishak M. Morbidity, mortality, and healthcare burden of nosocomial Clostridium difficile-associated diarrhea in Canadaian hospitals. Infect Control Hosp Epidemiol. 2002;23(3):137-140.

39. Ministry of Helath and Long-Term Care. Ontario health planning data guide - release 3.0. 2006. Available from: https://www.health.gov.on.ca/ transformation/providers/information/resources/healthplan_guide.pdf. Accessed June 12, 2015.

40. Canadian Institute for Health Information. The cost of acute care hospital stays by medical condition in Cananda, 2004-2005. Ottawa: CIHI; 2008. Available from: https://secure.cihi.ca/free_products/ nhex_acutecare07_e.pdf. Accessed June 12, 2015. 


\section{Supplementary materials}

Table SI Diagnosis codes for identification of study population

\begin{tabular}{|c|c|}
\hline Description & ICD-I0-CA diagnosis code \\
\hline \multicolumn{2}{|l|}{ ABSSSI } \\
\hline Erysipelas & A46 \\
\hline Cellulitis, abscess, and other local infections of skin & L02.0-L02.9, L03.0-L03.9, L05.0, L08.0-L08.9 \\
\hline \multirow[t]{2}{*}{ Superficial injury, infected ${ }^{a}$} & Infection codes: L02.0-L02.9, L03.0-L03.9, \\
\hline & L05.0, L08.0-L08.9, and any injury site codes \\
\hline Face, neck, or scalp (except eye) & S00.x, SIO.x \\
\hline Thorax, abdomen, lower back, or pelvis & S20.x, S30.x \\
\hline Shoulder or arm & S40.x, S50.x \\
\hline Wrist or hand & S60.x \\
\hline Hip or thigh & S70.x, TI3.0, TI4.0 \\
\hline Knee or lower extremity & S80.x, S90.x \\
\hline Multiple or unspecified sites & T00.x \\
\hline \multicolumn{2}{|l|}{ MRSA } \\
\hline $\begin{array}{l}\text { Staphylococcus aureus as the cause of diseases classified } \\
\text { to other chapters }\end{array}$ & B95.6 \\
\hline Resistance to methicillin & U82.I B95.6 \\
\hline
\end{tabular}

Table S2 Ontario time trends

\begin{tabular}{|c|c|c|c|c|c|c|c|}
\hline \multirow[t]{2}{*}{ Characteristic } & \multicolumn{7}{|c|}{ Year of MRSA-positive ABSSSI acute care hospitalization } \\
\hline & 2008 & 2009 & 2010 & 2011 & 2012 & 2013 & 2014 \\
\hline \multicolumn{8}{|l|}{ ABSSSI primary diagnosis } \\
\hline Hospital LOS, days & $n=104$ & $\mathrm{n}=128$ & $\mathrm{n}=155$ & $n=152$ & $n=156$ & $\mathrm{n}=192$ & $\mathrm{n}=136$ \\
\hline Mean & 11.5 & 9.3 & 8.5 & 8.6 & 11.9 & 8.7 & 7.7 \\
\hline Standard deviation & 14.7 & 11.0 & 10.0 & 9.2 & 20.2 & 8.0 & 7.0 \\
\hline Special care facility LOS, hours & $n=4$ & $\mathrm{n}=5$ & $n=7$ & $\mathrm{n}=9$ & $\mathrm{n}=7$ & $\mathrm{n}=3$ & $\mathrm{n}=2$ \\
\hline Mean & 158.5 & 96.6 & |44.| & I74.| & 121.0 & 66.3 & 160.5 \\
\hline Standard deviation & 194.0 & 72.3 & 129.3 & 166.5 & 123.2 & 40.2 & 78.5 \\
\hline \multicolumn{8}{|l|}{ ABSSSI secondary diagnosis } \\
\hline Hospital LOS, days & $n=137$ & $n=128$ & $\mathrm{n}=155$ & $n=152$ & $n=156$ & $n=192$ & $\mathrm{n}=136$ \\
\hline Mean & 31.5 & 28.2 & 22.5 & 24.0 & 28.9 & 21.1 & 18.2 \\
\hline Standard deviation & 42.2 & 45.4 & 33.3 & 32.7 & 62.7 & 31.0 & 21.3 \\
\hline Special care facility LOS, hours & $n=23$ & $n=19$ & $n=16$ & $\mathrm{n}=25$ & $n=32$ & $n=31$ & $n=27$ \\
\hline Mean & 188.8 & 158.6 & 145.9 & 431.0 & 167.1 & 136.0 & 160.3 \\
\hline Standard deviation & 158.3 & 176.2 & 103.4 & 957.0 & 197.2 & 161.0 & 150.0 \\
\hline
\end{tabular}

Abbreviations: ABSSSI, acute bacterial skin structure tissue infections; LOS, length of stay; MRSA, methicillin-resistant Staphylococcus aureus. 
Table S3 Atlantic Canada time trends

\begin{tabular}{|c|c|c|c|c|c|c|c|}
\hline \multirow[t]{2}{*}{ Characteristic } & \multicolumn{7}{|c|}{ Year of MRSA-positive ABSSSI acute care hospitalization } \\
\hline & 2008 & 2009 & 2010 & 2011 & 2012 & 2013 & 2014 \\
\hline \multicolumn{8}{|l|}{ ABSSSI primary diagnosis } \\
\hline Hospital LOS, days & $n=25$ & $n=26$ & $n=25$ & $n=4 I$ & $n=18$ & $n=24$ & $n=14$ \\
\hline Mean & 14.0 & 24.7 & 11.5 & 30.6 & 10.4 & 12.5 & 8.9 \\
\hline Standard deviation & 13.2 & 67.0 & 9.6 & 64.5 & 11.8 & 16.5 & 10.8 \\
\hline Special care facility LOS, hours & $\mathrm{n}=2$ & $n=2$ & $n=1$ & $n=3$ & $\mathrm{n}=0$ & $n=I$ & $\mathrm{n}=0$ \\
\hline Mean & 53.5 & 251.5 & 24.0 & 100.0 & - & 61.0 & - \\
\hline Standard deviation & 47.4 & 221.3 & - & 136.0 & - & - & - \\
\hline \multicolumn{8}{|l|}{ ABSSSI secondary diagnosis } \\
\hline Hospital LOS, days & $n=|4|$ & $n=72$ & $\mathrm{n}=74$ & $n=48$ & $n=43$ & $\mathrm{n}=42$ & $n=26$ \\
\hline Mean & 47.6 & 40.5 & 51.8 & 32.9 & 35.1 & 42.1 & 25.2 \\
\hline Standard deviation & 98.5 & 59.8 & 155.2 & 32.8 & 53.9 & 57.3 & 35.6 \\
\hline Special care facility LOS, hours & $\mathrm{n}=14$ & $\mathrm{n}=8$ & $n=15$ & $n=12$ & $\mathrm{n}=6$ & $\mathrm{n}=4$ & $\mathrm{n}=6$ \\
\hline Mean & 150.9 & 278.3 & 153.3 & 119.4 & 324.8 & 471.0 & 169.5 \\
\hline Standard deviation & 118.9 & 468.1 & 135.4 & 115.9 & 442.4 & 611.3 & 235.4 \\
\hline
\end{tabular}

Abbreviations: ABSSSI, acute bacterial skin and skin structure infections; LOS, length of stay; MRSA, methicillin-resistant Staphylococcus aureus; “-”, no data.

Table S4 Alberta and British Columbia time trends

\begin{tabular}{|c|c|c|c|c|c|c|c|}
\hline \multirow[t]{2}{*}{ Characteristic } & \multicolumn{7}{|c|}{ Year of MRSA-positive ABSSSI acute care hospitalization } \\
\hline & 2008 & 2009 & 2010 & 2011 & 2012 & 2013 & 2014 \\
\hline \multicolumn{8}{|l|}{ ABSSSI primary diagnosis } \\
\hline Hospital LOS, days & $\mathrm{n}=240$ & $\mathrm{n}=205$ & $\mathrm{n}=238$ & $\mathrm{n}=234$ & $n=232$ & $n=24 I$ & $n=212$ \\
\hline Mean & 13.5 & 13.6 & 11.8 & 16.1 & 12.5 & 11.0 & 12.4 \\
\hline Standard deviation & 19.3 & 24.1 & 15.9 & 35.2 & 17.8 & 11.0 & 15.1 \\
\hline Special care facility LOS, hours & $\mathrm{n}=5$ & $\mathrm{n}=\mathrm{I}$ & $n=7$ & $\mathrm{n}=6$ & $n=4$ & $n=5$ & $\mathrm{n}=5$ \\
\hline Mean & 31.8 & 43.0 & 113.4 & 85.5 & 133.3 & 85.2 & 57.2 \\
\hline Standard deviation & 23.3 & - & 68.1 & 75.5 & 68.3 & 86.2 & 44.7 \\
\hline \multicolumn{8}{|l|}{ ABSSSI secondary diagnosis } \\
\hline Hospital LOS, days & $\mathrm{n}=224$ & $\mathrm{n}=236$ & $\mathrm{n}=247$ & $\mathrm{n}=249$ & $\mathrm{n}=245$ & $\mathrm{n}=260$ & $\mathrm{n}=209$ \\
\hline Mean & 37.1 & 41.2 & 41.5 & 33.9 & 28.6 & 26.0 & 25.0 \\
\hline Standard deviation & 79.7 & 76.4 & 88.6 & 48.9 & 39.7 & 34.2 & 32.1 \\
\hline Special care facility LOS, hours & $\mathrm{n}=20$ & $n=24$ & $\mathrm{n}=27$ & $n=23$ & $n=29$ & $n=26$ & $n=21$ \\
\hline Mean & 261.9 & 117.4 & 230.0 & I55.| & 179.9 & 120.9 & $|4| .5$ \\
\hline Standard deviation & 340.2 & 106.0 & 232.6 & 151.6 & 137.7 & 97.5 & 190.1 \\
\hline
\end{tabular}

Abbreviations: ABSSSI, acute bacterial skin and skin structure infections; LOS, length of stay; MRSA, methicillin-resistant Staphylococcus aureus; “-”, no data.

Table S5 Canadian Prairie time trends

\begin{tabular}{|c|c|c|c|c|c|c|c|}
\hline \multirow[t]{2}{*}{ Characteristic } & \multicolumn{7}{|c|}{ Year of MRSA-positive ABSSSI acute care hospitalization } \\
\hline & 2008 & 2009 & 2010 & 2011 & 2012 & 2013 & 2014 \\
\hline \multicolumn{8}{|l|}{ ABSSSI primary diagnosis } \\
\hline Hospital LOS, days & $\mathrm{n}=36$ & $\mathrm{n}=54$ & $\mathrm{n}=77$ & $\mathrm{n}=89$ & $\mathrm{n}=76$ & $\mathrm{n}=77$ & $\mathrm{n}=66$ \\
\hline Mean & 11.9 & 9.7 & 11.2 & 19.3 & 8.2 & 11.3 & 13.4 \\
\hline Standard deviation & 29.4 & II.I & 24.5 & 40.3 & 9.4 & 22.0 & 21.8 \\
\hline Special care facility LOS, hours & $\mathrm{n}=0$ & $\mathrm{n}=0$ & $\mathrm{n}=\mathrm{l}$ & $\mathrm{n}=2$ & $n=1$ & $\mathrm{n}=2$ & $n=2$ \\
\hline Mean & - & - & 22.0 & 121.5 & 48.0 & 32.5 & 438.5 \\
\hline Standard deviation & - & - & - & 128.0 & - & 20.5 & 593.3 \\
\hline \multicolumn{8}{|l|}{ ABSSSI secondary diagnosis } \\
\hline Hospital LOS, days & $\mathrm{n}=20$ & $n=43$ & $\mathrm{n}=60$ & $\mathrm{n}=65$ & $n=67$ & $\mathrm{n}=70$ & $n=52$ \\
\hline Mean & 48.5 & 34.6 & 19.5 & 39.6 & 40.1 & 24.8 & 18.3 \\
\hline Standard deviation & 73.0 & 50.9 & 29.3 & 65.8 & 77.5 & 40.8 & 18.1 \\
\hline Special care facility LOS, hours & $\mathrm{n}=4$ & $\mathrm{n}=8$ & $\mathrm{n}=8$ & $n=12$ & $n=12$ & $n=12$ & $n=6$ \\
\hline Mean & 211.0 & 246.9 & 195.3 & 134.1 & 127.1 & 118.6 & 92.3 \\
\hline Standard deviation & 282.9 & 334.8 & 149.0 & 90.2 & 104.8 & 82.9 & 47.6 \\
\hline
\end{tabular}

Abbreviations: ABSSSI, acute bacterial skin and skin structure infections; LOS, length of stay; MRSA, methicillin-resistant Staphylococcus aureus; “-”, no data. 
Infection and Drug Resistance

\section{Publish your work in this journal}

Infection and Drug Resistance is an international, peer-reviewed openaccess journal that focuses on the optimal treatment of infection (bacterial, fungal and viral) and the development and institution of preventive strategies to minimize the development and spread of resistance. The journal is specifically concerned with the epidemiology of antibiotic

resistance and the mechanisms of resistance development and diffusion in both hospitals and the community. The manuscript management system is completely online and includes a very quick and fair peerreview system, which is all easy to use. Visit http://www.dovepress.com/ testimonials.php to read real quotes from published authors.

Submit your manuscript here: http://www.dovepress.com/infection-and-drug-resistance-journal 